\title{
Error and Noise Analysis in an IMU using Kalman Filter
}

\author{
S.A.Quadri and Othman Sidek \\ Collaborative Microelectronic Design Excellence Centre (CEDEC) \\ Universiti Sains Malaysia, Engineering Campus, Nibong Tebal \\ reachquadri@yahoo.com
}

\begin{abstract}
Kalman filtering is a well-established methodology used in various multi-sensor data fusion applications. In our experiment, we first obtain measurements from the acclerometer and gyroscope and fuse them using Kalman filter in an inertial measurement uni (MMU). We estimate Kalman filter output and estimation error. The affect of process noise and measurement noise on estimation error is tested. It is explored that the neasurement noise has significant role to increase estimation error in the datafusion process.
\end{abstract}

Keywords: Kalman filter, Data fusion, Accelerometer, Gyroscope, Process noise, Inertial measurement unit, Measurement noise

\section{Introduction}

In recent years, with the developmentof sensor technology and processing algorithms, multi-sensor data fusion has receivedsignificant attention in many engineering applications. Data fusion plays a critical and fundamental oly in achieving accuracy and precision. For many applications, accuracy and precision are key performance metrics.

One of the main concerns in any fusion technique is the risk of producing a fused system result that is actually performing worse than the best individual tool. Poor estimation could be responsible for poof performance of data fusion system. The paper presents fusion of two estimates from gyoscope and accelerometer in an IMU employing Kalman filter, which is well-known optimal estimator. The noise and other inaccuracies contribute to estimation error in fusion process. This brief paper presents the effect of measurement noise and process noise on the estimation error

\section{Inertial Measurement Unit}

An inertial measurement unit (IMU) is an electronic device that measures and reports on a craft's velocity, orientation, and gravitational forces, using a combination of accelerometers and gyroscopes. IMUs are typically used to maneuver aircraft, including unmanned aerial vehicles (UAV), spacecraft, including shuttles and satellites. An IMU works by detecting the current rate of acceleration using one or more accelerometers, and detects changes in rotational attributes like pitch, roll and yaw using one or more gyroscopes. A basic unit with assembly of components is shown in Figure 1. A detail description of principle, working and application of IMU could be found in [1-3]. 


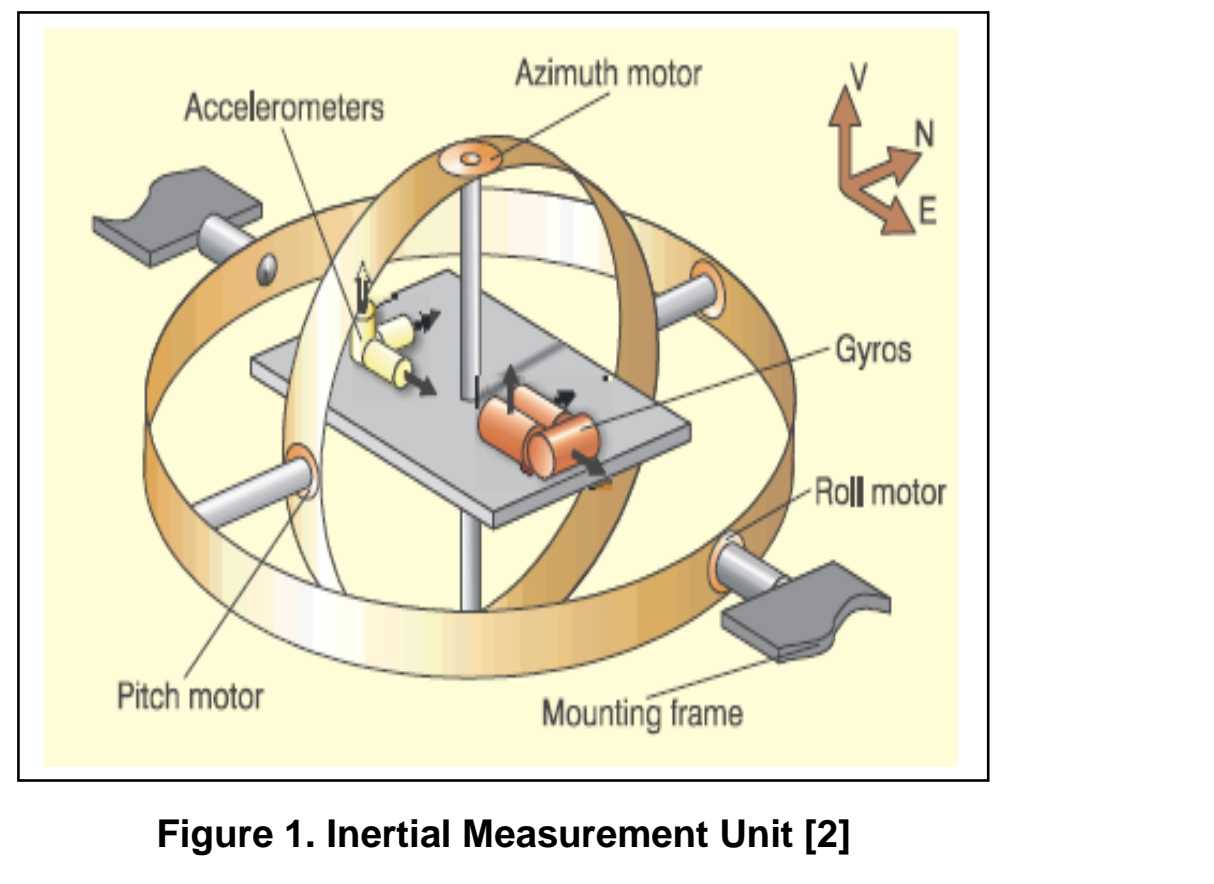

An ideal MEMS gyroscope produces a predictable output when it is subjected to a known rate of rotation. It has no noise, perfect linearity, and no offset, however, that is not possible to achieve practically. Mark Looney [1] discussed many factors on which the performance of gyroscope depends such as bias effeet, noise, scale factor error and displacementmeasurement errors. King [2] discussed many errors encountered during practical usage of gyroscope such as initial tilt error, gyro drifterorgand azimuth gyro drifts.

MEMS gyroscope and accelerometers have many real time applications. With the implementation of Kalman fotters, wavelet transforms and neural networks the occurrence of drift in the gyroscope time series has been significantly reduced [4-5]. Although gyroscope and accelerometer are associated with many errors as stated above, in this brief paper the authors have focused only on estimation error, which is encountered using Kalman filter technique in the data fusion process.

\section{Kalman Filter and Rélated Work}

The traditional Kamman filter requires exact knowledge of the plant model and the statistics of the process noise and measurement noise. Kalman filtering with unknown process noise and measurement noise covariances is a classical problem [6]. Varieties of methods have been developed to simultaneously estimate the covariances with the state [7-8]. Measurement noise is well knowh at all times, the process noise is unknown in real time, but can be inferred from the measurements (which are affected by measurement noise) [9-10].

Sasa [11] discussed the robustness of a Kalman filter against uncertainties of noise covariances. When covariance matrices of process noise and observation noise change from their nominal levels multiplied by random variables, a Kalman filter designed for the nominal noise condition is shown to be more robust than an observer designed by the pole placement technique in terms of the amount of deviation of the estimation accuracy from a nominal value. 
Garcia-Velo [12] provided a methodology for estimating an appropriate process noise covariance, based on the recursive minimization of the difference between residual covariance matrix given by the filter and the observed residual sequence.

There have been many investigations in the area of adaptive filter. Maybeck [13] used a maximum-likelihood estimator to estimate the system errors covariance matrix. Lee and Alfriend [14] modified the above methods by introducing a window scale factor. Loebis et al. [15] presented an adaptive Extended Kalman filter (EKF) method, which adjusts the measurement noise covariance matrix by fuzzy logic technique. Han and $\mathrm{Wu}$ [16] proposed a new adaptive Unscented Kalman Filter (UKF) algorithm for actuator failure estimation in rotorcraft unmanned aerial vehicle (RUAV) application. The filter method with adaptability to statistical characteristic of noise was presented to improve the estimation accuracy of traditional UKF. Adaptive mechanism was anticipated to compensate the lack of a prior knowledge.

Shi et al. [17] derived a new performance-bound for a sensor fusion schemethat explicitly takes the model uncertainty of the underlying processes and sensors into account Based on the classical Kalman filter, the estimation error covariance is computed for given uncertainties of the process and measurement noise covarjance. This/approach was implemented in multi sensor network application.

Wang [18] presented an improved adaptive Kalman filter algorithm to model error and process noise uncertainty in global positioning system (GPS) relative application. The adaptive algorithm to model error was obtained by using an upper bound for the state prediction covariance matrix. The process noise was estimated at each filter step by minimizing a criterion function, which-was determined by measurement prediction. A recursive algorithm was provided for solving the criterion function.

Our present study is focused on performance of data fusion in IMU using Kalman filter technique, exploring the effect of process noise and measurement noise on estimation error. Under simulation environment in has been investigated that introduction of measurement noise has a significant role foincrease the estimation error.

\section{The State Estimation Problem}

The Discrete time linear systemsare often represented in a state variable format given by the equation:

$$
\mathrm{x}_{\mathrm{j}}=\mathrm{ax}_{\mathrm{j}-1}+\mathrm{bu}_{\mathrm{j}} /(1)
$$

Where the state $x_{j}$ is a scalar, a and $\mathrm{b}$ are constants and the input $u_{j}$ is a scalar; $j$ represents the time variable. We use the variable $\mathrm{k}$ to represents Kalman filter gain and $\mathrm{T}$ to time delay. Suppose some noise is added to the process such that:

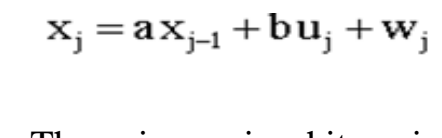

The noise, $w$, is white noise source with zero mean and covariance Q and is uncorrelated with the input. The process can now be represented as shown below,

We filter the signal $\mathrm{x}$ to minimize the effect of noise $\mathrm{w}$. Let us assume that the signal $\mathrm{x}$ is not directly measured, but instead we measure $\mathrm{z}$.

$$
\mathrm{z}_{\mathrm{j}}=\mathrm{hx}_{\mathrm{j}}+\mathrm{v}_{\mathrm{j}}
$$


The measured value $\mathrm{z}$ depends on the current value of $\mathrm{x}$, as determined by the gain $h$. Additionally, the measurement has its own noise, $v$, associated with it. The noise, $v$, is white noise source with zero mean and covariance $\mathrm{R}$ that is uncorrelated with the input or with the noise $w$. The two noise sources are independent of each other and independent of the input. The task of the Kalman filter is to filter $z$ to estimate the variable $\mathrm{x}$ while minimizing the effects of $w$ and $v$. It seems reasonable to achieve an estimate of the state (and the output) by simply reproducing the system architecture. This simple way to get an estimate of $\mathrm{x}_{\mathrm{j}}$ (which we will call $\mathrm{x}^{\wedge} \mathrm{j}$, a priori estimate), is diagrammed in Figure 2 [19].

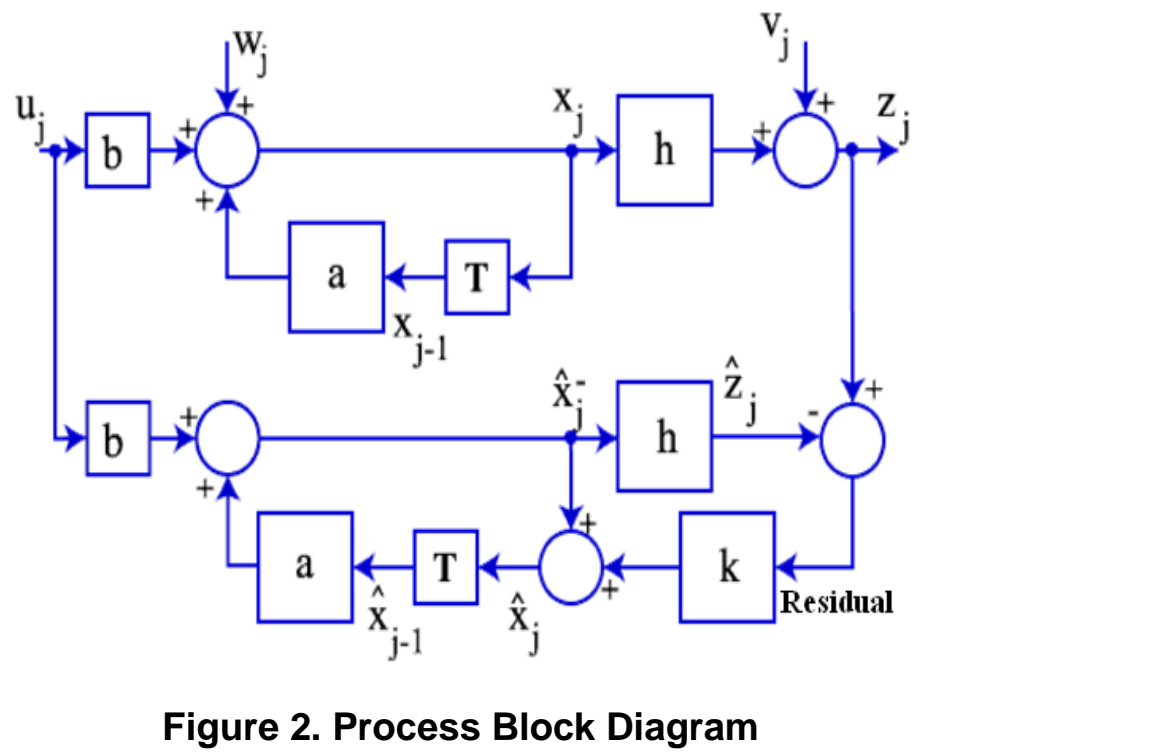

Figure 2. shows correction mechanism as well as compensation for addition of noise source ( $\mathrm{w}$ and $\mathrm{v})$,

$$
\hat{\mathrm{x}}_{\mathrm{j}}^{-}=\mathbf{a} \hat{\mathrm{x}}_{\mathrm{j}-1}+\mathrm{bu}_{\mathrm{j}}
$$

We use this a priori es imate to predict an estimate for the output, $\mathrm{z}^{\wedge} \mathrm{j}$. The difference between this estimated output and the actual output is called the residual, or innovation.

$$
\text { Residual }=\mathrm{z}_{\mathrm{j}}-\hat{z}_{\mathrm{j}}=\mathrm{z}_{\mathrm{j}}-\mathrm{h} \hat{\mathrm{x}}_{\mathrm{j}}^{-}
$$

If the residual is small, it generally means we have a good estimate; if it is large, the estimate is not so good. We can use this information to refine our estimate of $x_{j}$; we call this new estimate the a posteriori estimate, $\mathrm{x}^{\wedge} \mathrm{j}$.. If the residual is small, so is the correction to the estimate. As the residual grows, so does the correction. The pertinent equation is (from the Figure 2).

$$
\hat{\mathrm{x}}_{\mathrm{j}}=\hat{\mathrm{x}}_{\mathrm{j}}^{-}+\mathrm{k}(\text { Residual })=\hat{\mathrm{x}}_{\mathrm{j}}^{-}+\mathrm{k}\left(\mathrm{z}_{\mathrm{j}}-\mathrm{h} \hat{\mathrm{x}}_{\mathrm{j}}^{-}\right)
$$

The only task now is to find the quantity $\mathrm{k}$ that is used to refine our estimate, and it is this process that is at the heart of Kalman filtering. 
To begin, let us define the errors of our estimate. There will be two errors, an a priori error, $e_{j}{ }_{j}$, and an a posteriori error, $e_{j}$. Each one is defined as the difference between the actual value of $\mathrm{x}_{\mathrm{j}}$ and the estimate (either a priori or a posteriori).

$$
\begin{aligned}
& e_{j}^{-}=x_{j}-\hat{x}_{j}^{-} \\
& e_{j}=x_{j}-\hat{x}_{j}
\end{aligned}
$$

Associated with each of these errors is a mean squared error, or variance:

$$
\begin{aligned}
& \mathbf{p}_{j}^{-}=\mathbf{E}\left\{\left(\mathbf{e}_{j}^{-}\right)^{2}\right\} \\
& \mathbf{p}_{j}=\mathbf{E}\left\{\left(\mathbf{e}_{j}\right)^{2}\right\}
\end{aligned}
$$

Where the operator $\mathrm{E}\{\}$ represents the expected, or average, value. A Kalman filter minimizes the a posteriori variance, $\mathrm{p}_{\mathrm{j}}$, by suitably choosing the value of $\mathrm{k}$. We starl by substituting equation 7 into equation 8 , and then substituting in equation 6 .

$$
\begin{aligned}
& \mathrm{p}_{\mathrm{j}}=\mathrm{E}\left\{\left(\mathrm{x}_{\mathrm{j}}-\hat{\mathrm{x}}_{\mathrm{j}}\right)^{2}\right\} \\
& \mathrm{p}_{\mathrm{j}}=\mathrm{E}\left\{\left(\mathrm{x}_{\mathrm{j}}-\hat{\mathrm{x}}_{\mathrm{j}}^{-}-\mathrm{k}\left(\mathrm{z}_{\mathrm{j}}-\mathrm{h} \hat{\mathrm{x}}_{\mathrm{j}}^{-}\right)\right)^{2}\right\}
\end{aligned}
$$

To find the value of $\mathrm{k}$ that minimizes the variance we differentiate this expression with respect to $\mathrm{k}$ and set the derivative to zero.

It can be shown for Kalman gain [19],

$$
\mathbf{k}=\frac{\mathbf{h} \mathbf{p}_{j}^{-}}{\mathbf{h}^{2} \mathbf{p}_{j}^{-}+\mathbf{R}}
$$

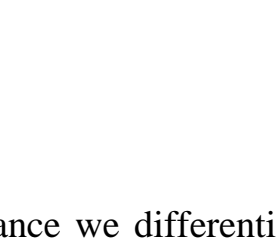

Any Kalman filter operation begins with a system description consisting of gains a, b and $\mathrm{h}$. The state is $\mathrm{x}$, the input to the system is $\mathrm{u}$ and the output is $\mathrm{z}$. The time index is given by $\mathrm{j}$.

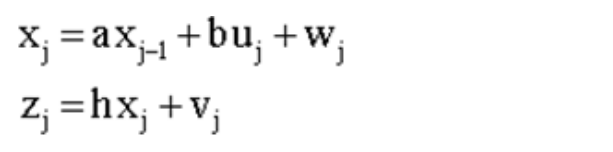

The process has two steps, a predictor step (which calculates the next estimate of the state based only on past measurements of the output), and a corrector step (which uses the current value of the estimate to (efine the result given by the predictor step).

In predictor step, we form the a priori state estimate based on the previous estimate of the state and the current value of the input.

$$
\hat{x}_{j}^{-}=a \hat{x}_{j}+b u_{j}
$$

We can calculate the a priori covariance [19]

$$
\mathrm{p}_{\mathrm{j}}^{-}=\mathrm{a}^{2} \mathrm{p}_{\mathrm{j}-1}+\mathrm{Q}
$$

Note that these two equations use previous values of the a posteriori state estimate and covariance. Therefore the first iteration of a Kalman filter requires estimates (which are often just guesses) of these two variables. The exact estimate is often not important as the values 
converge towards the correct value over time; a bad initial estimate just takes more iteration to converge.

In corrector step, to correct the a priori estimate, we need the Kalman filter gain, $\mathrm{k}$.

$$
\mathrm{k}_{\mathrm{j}}=\frac{\mathrm{hp} \mathrm{p}_{\mathrm{j}}^{-}}{\mathrm{h}^{2} \mathrm{p}_{\mathrm{j}}^{-}+\mathrm{R}}
$$

This gain is used to refine (correct) the a priori estimate to give us the a posteriori estimates.

$$
\hat{\mathrm{x}}_{\mathrm{j}}=\hat{\mathrm{x}}_{\mathrm{j}}^{-}+\mathrm{k}_{\mathrm{j}}\left(\mathrm{z}_{\mathrm{j}}-\mathrm{h} \hat{\mathrm{x}}_{\mathrm{j}}^{-}\right)
$$

We can calculate the a posteriori covariance [19]

$$
\mathrm{p}_{\mathrm{j}}=\mathrm{p}_{\mathrm{j}}^{-}\left(1-\mathrm{hk}_{\mathrm{j}}\right)
$$

If the a priori error is very small, $\mathrm{k}$ is correspondingly very small, so out correction is also very small. In other words, we will ignore the current measurement and simply use past estimates to form the new estimate. This is as expected, if our first estimate (the a priori estimate) is good (i.e., with small error) there is very little needto correct it.

If the a priori error is very large (so that the measurement noise term, $\mathrm{R}$, in the denominator is unimportant) then $\mathrm{k}=1 / \mathrm{h}$. This, in effect (teils us to use the current (measured) value of the output to estimate the stater. It the a ptioni error is large then we should disregard the a priori estimate, and instead use the current measurement of the output to form our estimate of the state.

$$
\begin{aligned}
\hat{\mathrm{x}}_{\mathrm{j}} & =\hat{\mathrm{x}}_{\mathrm{j}}^{-}+\mathrm{k}(\text { Residual })=\hat{\mathrm{x}}_{\mathrm{j}}^{-}+\mathrm{k}\left(2-\mathrm{h} \hat{\mathrm{x}}_{\mathrm{j}}^{-}\right) \\
& =\hat{\mathrm{x}}_{\mathrm{j}}^{-}+\frac{1}{\mathrm{~h}}\left(\mathrm{z}_{\mathrm{j}}-\mathrm{h} \hat{\mathrm{x}}^{-}\right. \\
& =\frac{\mathrm{z}_{\mathrm{j}}}{\mathrm{h}}
\end{aligned}
$$

If the measurement noise, $\mathrm{R}$, is very large, $\mathrm{k}$ is very small, thus low confidence in the measurement increases estimation error.

\section{Results and Discussion}

The gyroscope and accelerometer data is obtained directly from the SparkFun IMU having 5 Degrees of Freedom. Data read from 1000 samples of the accelerometer had a variance of 0.07701688 and gyroscope had a variance of 0.00025556 [20]. Measurement noise and process noise are plugged in the program code based on [12] and [19]. Data obtained from accelerometer and gyroscope and their fusion using Kalman filter is shown in Figure 3. Kalman filter output and estimation error is shown in Figure 4. 


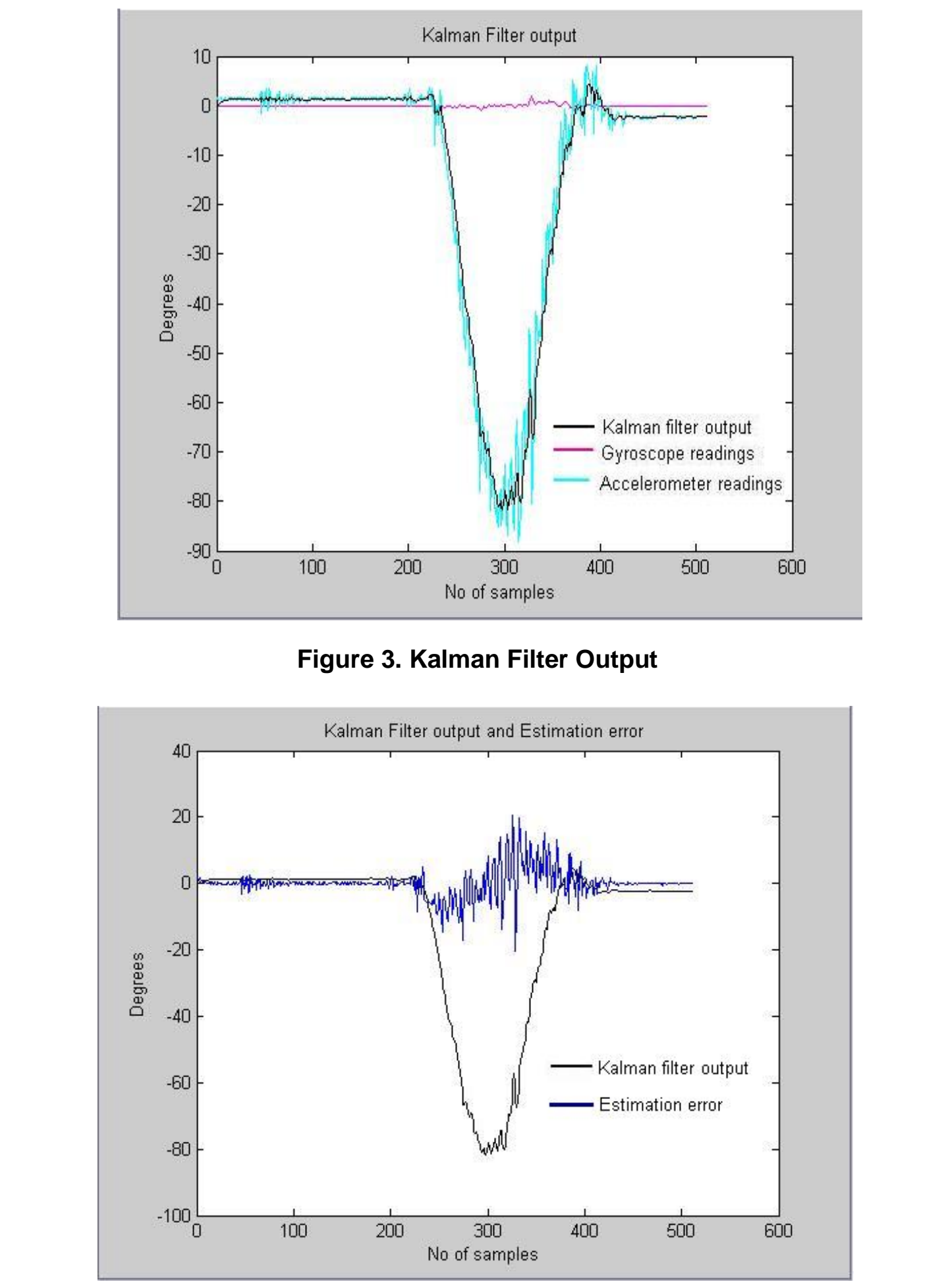

Figure 4. Kalman Filter Output and Estimation Error 
In order to compare the effect of process noise and measurement noise we consider two cases: Case 1) Plugging process noise and measurement noise is kept zero. Figure 5 shows the effect of the process noise on the estimation error.

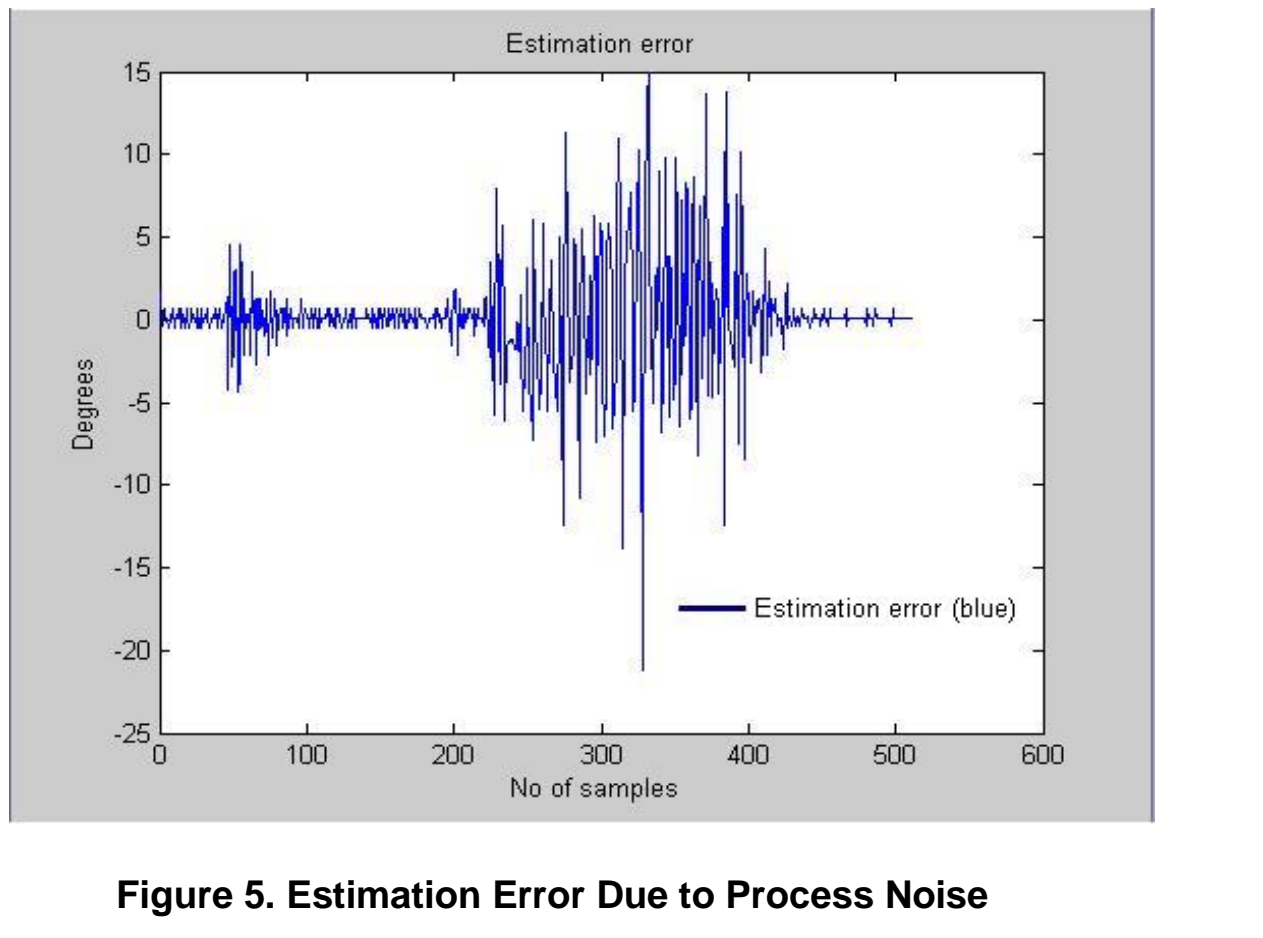

Case 2) Plugging the measurement noise and process noise is kept zero. Figure 6. shows effect of measurement noise on the estimation error.

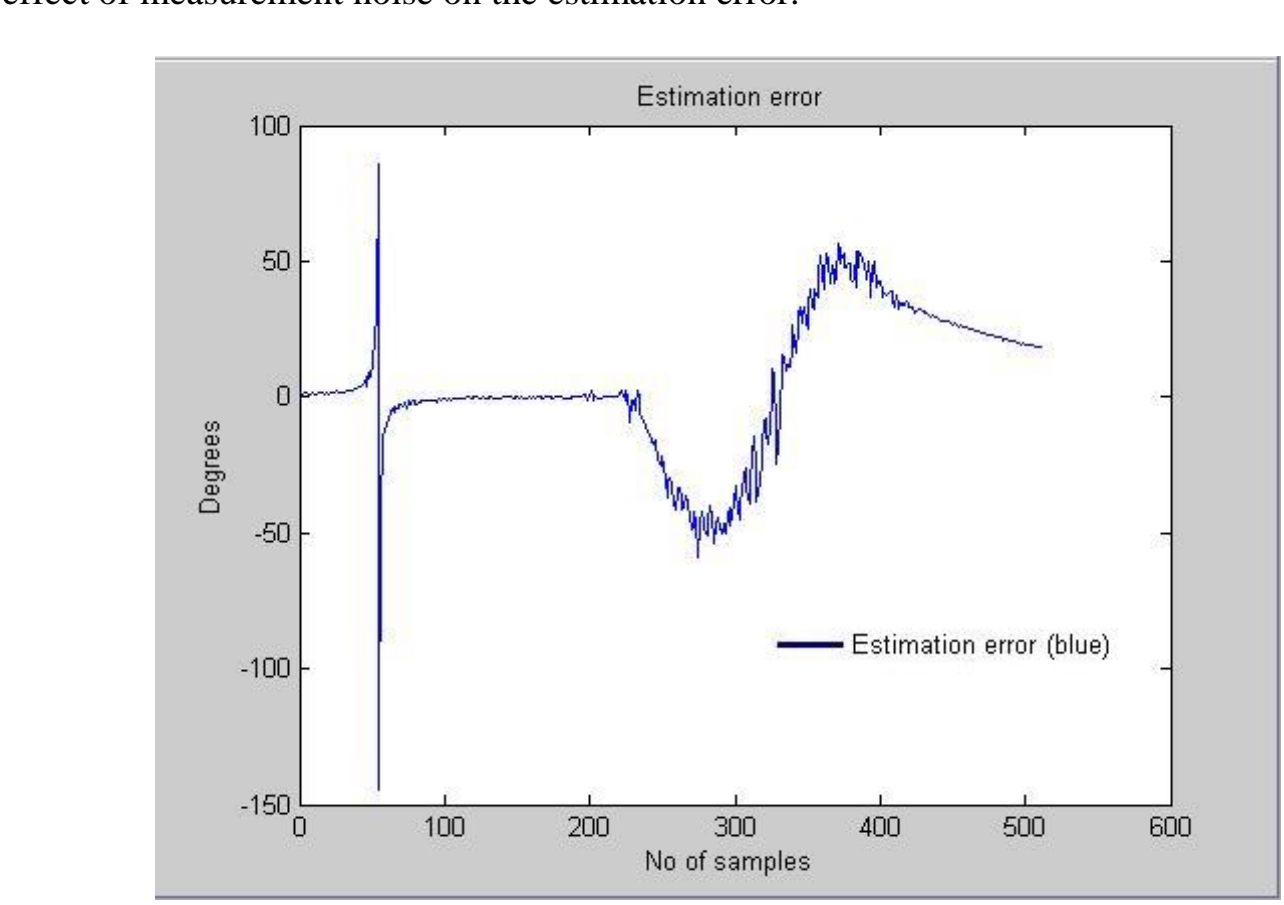

Figure 6. Estimation Error due to Measurement Noise 
In order to compare effects of both noises precisely, both the cases (case1 and case2) are plotted using least square method (LSM) [21] as shown in Figure 7.

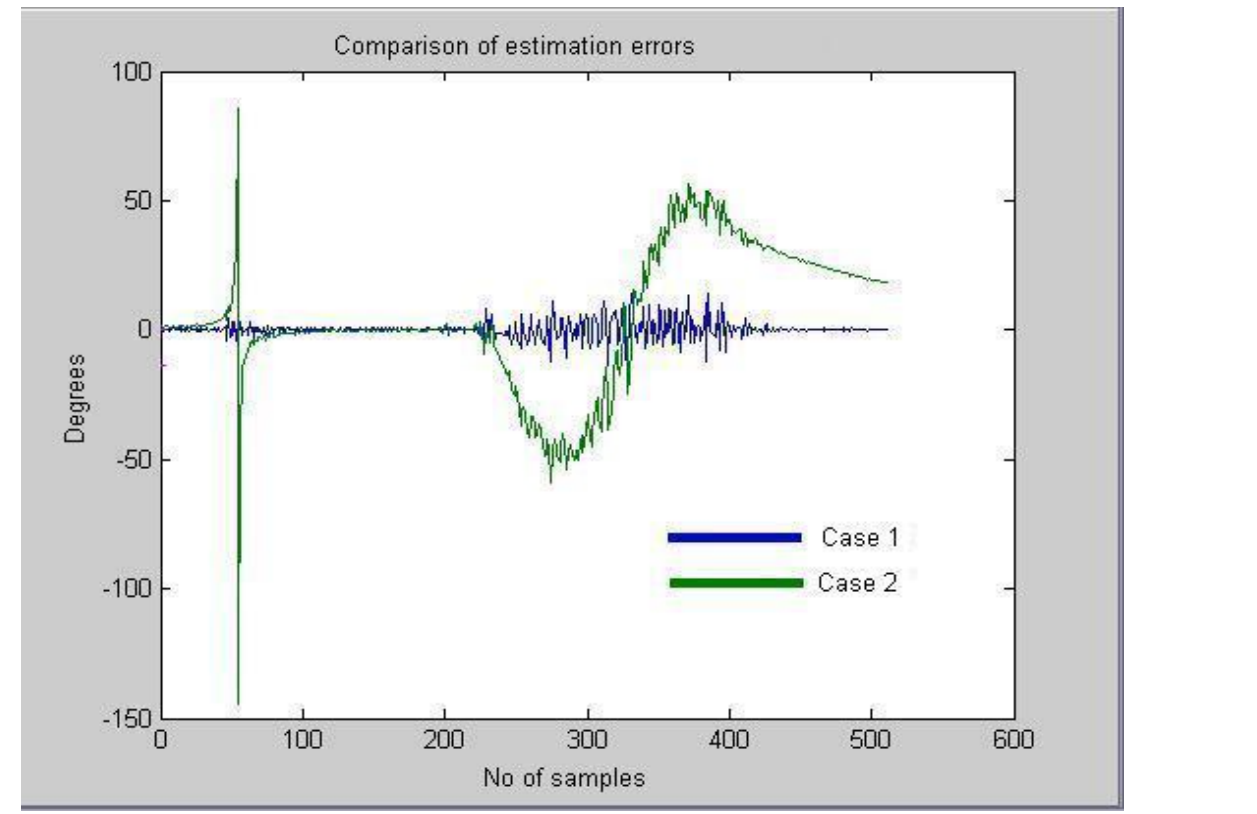

Figure 7. Estimation Êrrors Comparison using LSM

As discussed above in the estimation analysis, the increase in the measurement noise decreases value of the $\mathrm{k}$, consequently increasing the estimation error. Figure 7 evidently shows the effect of measurement noise is promment to increase the estimation error.

\section{Conclusion}

Estimating noise statistics is of great interest when using state-space models. Obviously, introduction of noise has its own futile affects. Exploring the factors that affect on data fusion performance is an active research area. Kalman filtering technique is employed to fuse data obtained from gyroscope and accelerometer in an IMU. Simulations are carried out to find the effect of measurement noise and process noise on estimation error. Finally, it is explored that measurement noise has greater impact on the estimation error as compared to the process noise.

\section{References}

[1] L. Mark, "A simple calibration for MEMS gyroscopes", EDN EUROPE Magazine, http://y ww.analog.com/static/imported-files/tech_articles/GyroCalibration_EDN_EU_7_2010.pdf, accessed (2014) April.

[2] A. D. King, "Inertial Navigation-Forty Years of Evolution", GEC Review, vol. 13, no. 3, (1998), pp. 140149.

[3] S. A. Quadri and O. Sidek, "Factors Affecting Data Fusion Performance in an Inertial Measurement Unit", Journal of Control Engineering and Technology (JCET), vol. 3 no. 3, (2013), pp. 107-110.

[4] X. Ji, S. Wang, Y. Xu, Q. Shi and D. Xia, "Application of the digital signal processing in the MEMS gyroscope de-drift", Proceedings of 1st IEEE international conf Nano/Micro Engineered and Molecular Systems, China, (2006), pp.218-221.

[5] R. C. Wagenaar and R. E. Emmerik, "Resonant frequencies of arms and legs identify different walking patterns", Journal of Biomechanics. vol. 33, no. 7, (2000), pp. $853-861$. 
[6] R. K. Mehra, "On the identification of variances and adaptive Kalman filtering", IEEE Transaction on Automatic Control, vol.15, no. 2, (1970), pp 175-184.

[7] T. Kailath, A. Sayed and B. Hassibi, "Linear Estimation", Prentice Hall, New Jersey, (2000), pp. 111-132.

[8] D. Simon, "Kalman filtering with state constraints: a survey of linear and nonlinear algorithms", IET Control Theory and Applications, vol. 4, no. 8, (2010), pp. 1303-1318.

[9] D. Simon, "Optimal state estimation, John Wiley and sons, New Jersey, (2006).

[10] F. Giri and E. Bai, "Block oriented Non linear system identification", Lecture notes in control and information sciences, Springer - Verlag Berlin Heidelberg, (2010), pp. 91-101.

[11] S. Sasa, "Robustness of a Kalman Filter against Uncertainties of Noise Covariances", Proceedings of American Control Conference, Philadelphia, Pennsylvania, (1998), pp. 2344-2348.

[12] J. B. Garcia-Velo, "Determination of noise covariances for extended Kalman filter parameter estimators to account for modeling errors", $\mathrm{PhD}$ thesis, Department of Aerospace Engineering and Engineering Mechanics of the College of Engineering, University of Cincinnati, Cincinnati, USA, (1997).

[13] P. Maybeck, "Stochastic Models, Estimation, and Control, Academic Press, New York, (1972).

[14] D. J. Lee and K. T. Alfriend, "Adaptive Sigma Point Filtering for State and Parameter Estimation", Proceedings of AIAA/AAS Astrodynamics Specialist Conference and Exhibition, Rhode Island, (200s), pp. $1-20$.

[15] D. Loebis, "Adaptive tuning of a Kalman filter via fuzzy logic for an intelligent AUV navigation system", Control Engineering and Practice, vol. 12, no. 12, (2004), pp. 1531-1539.

[16] J. Q. Han and Z. Wu, "Rotorcraft UAV Actuator Failure Estimation with KF-based Adaptive UKF Algorithm", Proceedings of American Control Conference, Westin Seattle Hotel, Seattle, Washington, USA, (2008), pp. 1618 - 1623.

[17] L. Shi, K. H. Johansson and R. M. Murray, "Kalman Filtering with Uncertain Process and Measurement Noise Covariances with Application to State Estimation in Sensor Networks , Proceedings of 16th IEEE International Conference Control Applications, Singapore, (2007), pp 1031-1036.

[18] X. Wang, "Improved adaptive filter with application to relative nâvigation", GPS Solutions, vol. 15, no. 2, (2011), pp. 121-128.

[19] http://www.swarthmore.edu/NatSci/echeeve1/Rer/Kalman/ScalarKalman.html (accessed (2014) April).

[20] http://home.comcast.net/ michael.p.thompson/kalman/kal man test2.c (accessed (2014) April).

[21] http://en.wikipedia.org/wiki/Least_squares (accessed (2014) April).
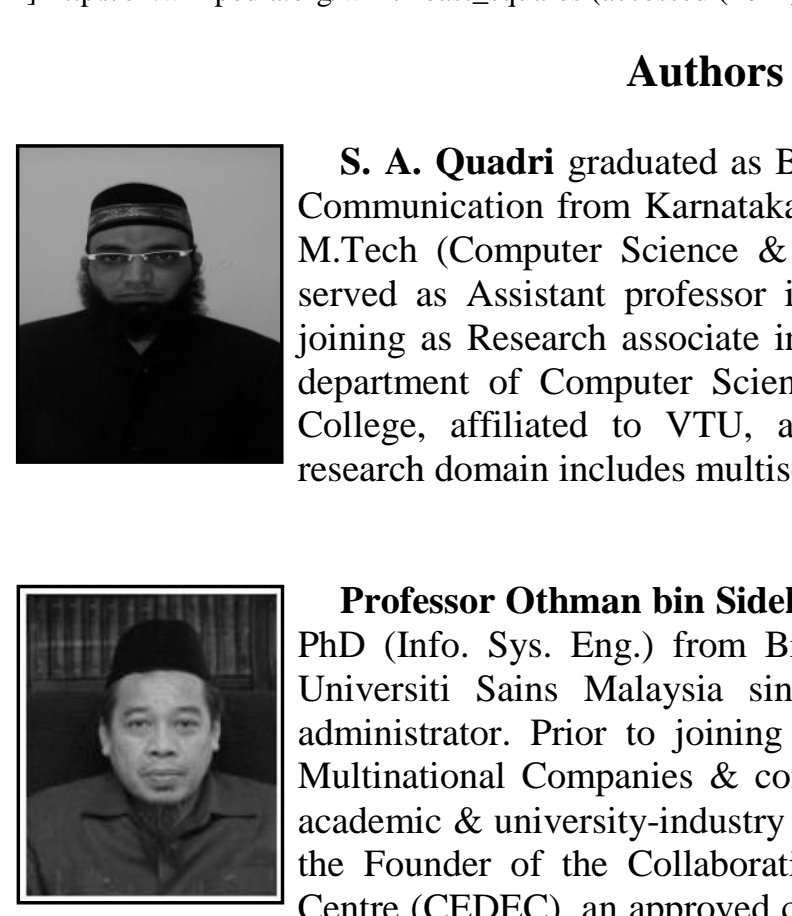

S. AQuadri graduated as Bachelor of Engineering in Electronics \& Cormunication from Karnataka University Dharwad. He completed his MTech (Computer Science \& Engineering) from VTU Belgaum. He served as Assistant professor in various Engineering colleges. Before joining as Research associate in CEDEC he served 3 years as Head of depatment of Computer Science \& Engineering department in SIET College, affiliated to VTU, approved by AICTE Delhi, India. His research domain includes multisensor data fusion \& neural networks.

Professor Othman bin Sidek an esteemed Malaysian scientist holds a PhD (Info. Sys. Eng.) from Bradford University, UK. He has served Universiti Sains Malaysia since 1984 as a lecturer, researcher, \& administrator. Prior to joining USM, he was a trainee engineer with Multinational Companies \& continued to take very active role in both academic \& university-industry research collaborations. Professor is also the Founder of the Collaborative Micro-electronic Design Excellence Centre (CEDEC), an approved centre at USM by the Malaysian Ministry of Finance in 2005 out of his own initiative. 